\title{
Correction to: Higher thyroid hormone levels and cancer
}

\author{
Petra Petranović Ovčariček ${ }^{1,2}$ (D) • Frederik A. Verburg ${ }^{1,3}$ - Martha Hoffmann ${ }^{1,4}$ • Ioannis lakovou ${ }^{1,5}$. \\ Jasna Mihailovic ${ }^{1,6,7}$. Alexis Vrachimis ${ }^{1,8}$. Markus Luster ${ }^{1,9} \cdot$ Luca Giovanella $^{1,10,11}$
}

Published online: 1 October 2020

(C) Springer-Verlag GmbH Germany, part of Springer Nature 2020

\author{
Correction to: Eur J Nucl Med Mol Imaging \\ https://doi.org/10.1007/s00259-020-05018-Z
}

This article is part of the Topical Collection on Erratum.

The online version of the original article can be found at https://doi.org/ $10.1007 / \mathrm{s} 00259-020-05018-\mathrm{Z}$

Petra Petranović Ovčariček

p.petranovic@gmail.com

EANM Thyroid Committee, Vienna, Austria

2 Department of Oncology and Nuclear Medicine, University Hospital Center "Sestre milosrdnice", Zagreb, Croatia

3 Department of Radiology and Nuclear Medicine, Erasmus MC, Rotterdam, The Netherlands

4 Department of Nuclear Medicine, Radiology Centre, Vienna, Austria

5 Academic Department of Nuclear Medicine, Aristotle University, Thessaloniki, Greece

6 Department of Radiology, Faculty of Medicine, University of Novi Sad, Novi Sad, Serbia
Department of Nuclear Medicine, Oncology Institute of Vojvodina, Sremska Kamenica, Serbia

8 Department of Nuclear Medicine, German Oncology Center, University Hospital of the European University, Limassol, Cyprus

9 University Hospital Marburg, Department of Nuclear Medicine, Marburg, Germany

10 Clinic for Nuclear Medicine and Competence Centre for Thyroid Diseases, Imaging Institute of Southern Switzerland, Bellinzona, Switzerland

11 Clinic for Nuclear Medicine, University Hospital and University of Zurich, Zurich, Switzerland 
Table 1 is incorrect in the original article, the correct Table 1 is shown

\begin{tabular}{|c|c|c|c|}
\hline Cancer site & Authors & Year & Correlation \\
\hline \multirow[t]{6}{*}{ Cancer in general } & Hellevik et al. [6] & 2009 & Low TSH $(<0.50 \mathrm{mU} / \mathrm{L})$ - increased cancer risk \\
\hline & Khan et al. [5] & 2016 & Higher FT4 - positive correlation with cancer risk \\
\hline & Yeh et al. [19] & 2013 & Hyperthyroidism — positive correlation with cancer risk \\
\hline & Vadiveloo et al. [16] & 2011 & $\begin{array}{l}\text { Subclinical hyperthyroidism-no correlation with } \\
\text { cancer risk }\end{array}$ \\
\hline & Brandt et al. [20] & 2013 & Toxic nodular goiter-linked with increased cancer mortality \\
\hline & Ittermann et al. [17] & 2010 & Low serum TSH levels - no correlation with cancer mortality \\
\hline
\end{tabular}

Zhang et al. [18]

Breast cancer

Khan et al. [5]

Tosovic et al. [21]

Søgaard et al. [10]

Tosovic et al. [22]

Kim et al. [23]

Ditsch et al. [24]

Tosovic et al. [8]

Journy et al. [9]

Prostate cancer Hellevik et al. [6]

Chan et al. [25]

Lehrer et al. [26]

Lehrer et al. [27]

Ovčariček et al. [28]

Ovarian cancer

Ness et al. [29]

Minlikeeva et al. [30]
2012 FT4 levels - positive correlation with breast cancer risk

2014 FT3 - negative correlation with cancer mortality

2016 Higher FT4 levels - positive correlation with breast cancer risk

2016 Hyperthyroidism - positive correlation with breast cancer risk

2010 T3 - positive correlation with breast cancer risk

2019 Higher FT4 levels - positive correlation with breast cancer risk Higher reference range TSH levels — negative correlation with breast cancer risk

2010 FT3 and FT4 levels - highest in breast cancer patients compared to healthy controls and patients with benign breast tumors

2014 Third T3 tertile-positive correlation with tumors larger than $20 \mathrm{~mm}$

Third T3 tertile - positive correlation with lymph node metastases

Third T3 tertile - positive correlation with negative estrogen receptor status

Third T3 tertile-positive correlation with negative progesterone receptor status

2017 Hyperthyroidism in 60-plus-year-old women - positive correlation with breast cancer mortality

2009 Low TSH level $(<0.50 \mathrm{mU} / \mathrm{L})$ - increased prostate cancer risk

2017 Higher FT4 levels - positive correlation with prostate cancer risk

Higher TSH levels - Negative correlation with prostate cancer risk

2002 T3 levels - higher in prostate cancer patients

2001 T3 levels - positive correlation with prostate cancer risk category

2020 T3 levels — positive correlation with less differentiated prostate cancer (higher in grade groups 3-5, compared to 1-2), higher pT stage, and larger prostate tumor involvement

2000 Hyperthyroidism - positive correlation with ovarian cancer risk

2017 Hyperthyroidism detected within the 5 years of ovarian cancer diagnosis - positive correlation with mortality
Adjusted HR 1.34, 95\% CI

$1.06-1.69$

Adjusted HR 1.42, 95\% CI 1.12-1.79; adjusted $\mathrm{HR}^{1} 1.13$ 95\% CI 0.99-1.28

Adjusted HR: $1.20,95 \%$ CI 1.01-1.43, $p<0.05$

Adjusted HR 0.98, 95\% CI $0.66-1.46$

Adjusted HR 1.36, 95\% CI $1.06-1.75, p<0.05$

Adjusted $\mathrm{HR}^{2} 1.07,95 \% \mathrm{CI}$ 0.57-2.00;

Adjusted $\mathrm{HR}^{3} 0.81,95 \% \mathrm{CI}$ $0.11-5.85$

Adjusted HR 0.62, 95\% CI $0.45-0.85, p=0.001$

Adjusted HR 1.77, 95\% CI 1.10-2.84; Adjusted $\mathrm{HR}^{1} 1.14$, 95\% CI 0.82-1.58

Adjusted OR ${ }^{4} 1.40,95 \%$ CI $1.10-1.77$

SIR 1.11, 95\% CI 1.07-1.16; SIR $^{5}$ $1.13,95 \%$ CI $1.08-1.19$

Adjusted RR ${ }^{6} 1.87,95 \%$ CI $1.12-3.14$

Adjusted HR 1.98, 95\% CI $1.02-3.83$

Adjusted $\mathrm{HR}^{7} 0.68,95 \% \mathrm{CI}$ $0.55-0.84$

FT3 and FT4: $p^{8}<0.001$

FT3: $p=0.021$; fT4: $p^{9}=0.017$

Adjusted HR 3.17, 95\% CI $1.20-8.36$

Adjusted HR 4.53, 95\% CI $1.60-12.83$

Adjusted HR 3.52, 95\% CI $1.32-9.41$

Adjusted HR 3.52, 95\% CI $1.42-8.75$

Adjusted HR 2.04, CI 1.16-3.60

Adjusted HR 1.97, 95\% CI 1.04-3.76; Adjusted HR ${ }^{10} 2.60$ (1.36-4.99)

Adjusted HR 1.11 per increase of $1 \mathrm{pmol} / \mathrm{L}, 95 \%$ CI 1.03-1.19, $p=0.009$

Adjusted $\mathrm{HR}^{11} 0.70,95 \% \mathrm{CI}$ $0.55-0.90, p=0.005$

$p=0.048$

$p=0.011$

$p=0.047, p=0.047, p=0.002$, respectively

OR 1.8

Adjusted HR 1.94, 95\% CI 1.19-3.18 
Table 1 (continued)

\begin{tabular}{|c|c|c|c|c|}
\hline Cancer site & Authors & Year & Correlation & \\
\hline \multirow{2}{*}{$\begin{array}{l}\text { Gastroesophageal } \\
\text { cancer }\end{array}$} & Turkyilmaz et al. [31] & 2010 & Incidence of hyperthyroidism - higher in the esophageal cancer patients & $p<0.001$ \\
\hline & Puhr et al. [32] & 2020 & Higher FT4 levels — shorter survival of gastroesophageal cancer patients & $\operatorname{HR} 2.20, p=0.041$ \\
\hline Pancreatic cancer & Ko et al. [33] & 2007 & Hyperthyroidism—positive correlation with pancreatic cancer risk & $\mathrm{OR}=2.1,95 \%$ CI $1.0-4.2$ \\
\hline \multirow[t]{2}{*}{ Colorectal cancer } & Boursi et al. [34] & 2015 & Hyperthyroidism — positive correlation with colorectal cancer risk & $\begin{array}{l}\text { Adjusted OR } 1.21,95 \% \mathrm{CI} \\
\quad 1.08-1.36, p=0.001\end{array}$ \\
\hline & L’Heureux et al. [35] & 2019 & Hyperthyroidism - negative correlation with colon cancer risk & $\begin{array}{l}\text { Adjusted OR } 0.74 ; 95 \% \text { CI } \\
\quad 0.64-0.85, p<0.001\end{array}$ \\
\hline \multirow[t]{2}{*}{ Lung cancer } & Hellevik et al. [6] & 2009 & Low TSH $(<0.50 \mathrm{mU} / \mathrm{L})$ - increased lung cancer risk & $\begin{array}{l}\text { Adjusted HR } 2.34,95 \% \text { CI } \\
\quad 1.24-4.40 \text {; Adjusted } \mathrm{HR}^{10} 2.91, \\
\quad 95 \% \text { CI } 1.49-5.70\end{array}$ \\
\hline & Khan et al. [5] & 2016 & Higher FT4 - positive correlation with lung cancer risk & $\begin{array}{l}\text { Adjusted HR } 2.33,95 \% \text { CI } \\
\text { 1.39-3.92; Adjusted HR }{ }^{1} 1.79 \text {, } \\
95 \% \text { CI } 1.23-2.59\end{array}$ \\
\hline \multirow[t]{2}{*}{ Thyroid cancer } & Yeh et al. [19] & 2013 & Hyperthyroidism - positive correlation with thyroid cancer risk & $\begin{array}{l}\text { Adjusted HR } 6.803,95 \% \text { CI } \\
\quad 3.58-12.91, p<0.05\end{array}$ \\
\hline & Diessl et al. [36] & 2012 & $\begin{array}{l}\text { T3 levels - negative correlation with the survival of advanced DTC } \\
\text { patients }\end{array}$ & $p^{12}=0.001$ \\
\hline Acute leukemia & Ghalaut et al. [37] & 2012 & $\begin{array}{l}\text { FT3, FT4, T3, and T4 levels are higher, while TSH is lower in acute } \\
\text { leukemia patients }\end{array}$ & $p<0.05$ \\
\hline $\begin{array}{l}\text { Myelodysplastic } \\
\text { syndrome }\end{array}$ & Dalamaga et al. [7] & 2008 & $\begin{array}{l}\text { FT3 and FT4 levels are higher, while TSH levels were lower in patients } \\
\text { with myelodysplastic syndrome }\end{array}$ & $p<0.05$ \\
\hline
\end{tabular}

$H R$, hazard ratio; SIR, standardized incidence ratios; $O R$, odds ratio

${ }^{1}$ After the exclusion of thyroid-affecting medicines; the highest tertile, compared to the lowest

${ }^{2}$ In subclinical hyperthyroidism

${ }^{3}$ In overt hyperthyroidism

${ }^{4}$ FT4 levels above vs. those below the median

${ }^{5}$ Beyond 5 years of follow-up

${ }^{6}$ The adjusted RR for the women in the fourth T3 quartile, as compared to the first

${ }^{7}$ The highest TSH tertile in the reference values, compared to the lowest tertile

${ }^{8}$ Breast cancer patients compared to healthy controls

${ }^{9}$ Breast cancer patients compared to patients with benign breast tumors

${ }^{10}$ After the exclusion of the first 2 years of follow-up

${ }^{11}$ Per increase in TSH of $1 \mathrm{IU} / \mathrm{L}$

${ }^{12}$ FT3 levels below vs. above $7.0 \mathrm{pmol} / \mathrm{L}$

Publisher's note Springer Nature remains neutral with regard to jurisdictional claims in published maps and institutional affiliations. 